\title{
PARÁSITOS Y ENFERMEDADES DEL VENADO BURA (Odocoileus hemionus fuliginatus) EN LA PARTE NORTE DE LA SIERRA SAN PEDRO MÁRTIR, BAJA CALIFORNIA, MÉXICO
}

\author{
JOAQUÍN CONTRERAS ${ }^{1,2}$, ERIC MELLINK³ ${ }^{3}$ R MARTÍNEZ ${ }^{1}$ Y G. MEDINA ${ }^{4}$ \\ ${ }^{1}$ Facultad de Ciencias, Universidad Autónoma de Baja California Km. 103 carretera \\ Tijuana-Ensenada, Baja California. México. C.P. 22860 \\ ${ }^{2}$ Dirección actual: Calle San Luis \# 106, Fracc. Costa Azul, Ensenada, Baja California, \\ México C.P. 22890 \\ ${ }^{3}$ Departamento de Biología de la Conservación Centro de Investigación Científica y de \\ Educación Superior de Ensenada Carretera Tijuana-Ensenada Km. 107 Ensenada, Baja \\ California C.P. 22860 \\ ${ }^{4}$ Instituto de Investigaciones Veterinarias, Universidad Autónoma de Baja California \\ Mexicali, Baja California, México. C.P. 21280 \\ correo electrónico:emellink@gmail.com
}

\begin{abstract}
Resumen: Durante los años 2001, 2002 y 2003 colectamos muestras de ectoparásitos, tejido sanguíneo y excretas de venados cobrados por cazadores en el norte de la sierra San Pedro Mártir, Baja California. Se colectaron ectoparásitos de 15 venados, lográndose identificar a Ixodes scapularis, Rhipicephalus sanguineus, Dermacentor occidentalis, Dermacentor variabilis, Dermacentor albipictus, Lipoptena sp, Hypoderma lineatum, Linognathus vituli y una larva de especie no identificada de la familia Oestridae. No se detectaron evidencias de nemátodos endoparásitos. Se analizaron los sueros de 12 venados para detectar anticuerpos contra el virus de la enfermedad de lengua azul, anaplasmosis y brucelosis registrando seropositividad a las dos primeras, pero no a brucelosis.
\end{abstract}

Palabras clave: Venado bura, parásitos, enfermedades, enfermedad de lengua azul, anaplasmosis, brucelosis.

\begin{abstract}
We collected samples of ectoparasites, blood, and feces of mule deer killed by sport hunters in the northern part of the Sierra San Pedro Mártir, Baja California from 2001 to 2003. We identified ectoparasites on 15 deer, which had 9 species of parasitic arthropods: Ixodes scapularis, Rhipicephalus sanguineus, Dermacentor occidentalis, Dermacentor variabilis, Dermacentor albipictus, Lipoptena sp, Hypoderma lineatum, Linognathus vituli, and one Oestridae larvae of unidentified species. We did not find any endoparasites. We analyzed blood serum from 12 deer to detect blue tongue disease, anaplasmosis, and brucellosis, and we found seropositivity to the two first diseases, but not to brucellosis.
\end{abstract}

Key words: Mule deer, extoparasites, disease, blue tongue disease, anaplasmosis, brucellosis. 


\section{INTRODUCCIÓN}

En México se cuenta con poca información acerca de parásitos y enfermedades de los animales silvestres y sobre sus implicaciones para las actividades humanas. En Baja California existen estudios zoosanitarios solamente de borrego cimarrón (Ovis canadensis). En éstos se detectó seroprevalencia de enfermedades de riesgo para el hombre, como Leptospira sp. y el ectima contagioso, y para el ganado, como la enfermedad hemorrágica epizoótica (Colodner, 2001). En otro estudio, sobre borrego cimarrón, se determinó la presencia de Dermacentor variabilis (López, 1979), una garrapata que es peligrosa porque puede transmitir fiebre maculosa de las Montañas Rocosas (rickettsia), babesiosis, anaplasmosis, tularemia (Francisella tularensis), fiebre Q (Coxiella burnetii) y provocar parálisis de las garrapatas (Allan, 2001b). Se desconoce si estas enfermedades existen en Baja California.

En el noreste de México, en 1988 y 1989, se detectó seroprevalencia de arbovirus tales como el de la estomatitis vesicular de Indiana, encefalitis-mena equina Venezolana II, virus del Río Grande, y estomatitis vesicular de New Jersey así como al cocobacilo Francisella tularensis, principalmente en mamíferos pequeños, y anticuerpos contra Anaplasma marginale en un venado cola blanca (Odocoileus virginianus; Aguirre et al., 1992). Otro estudio reportó evidencias virológicas de enfermedad de lengua azul en ganado bovino de Baja California (Stott et al., 1989).

En contraparte con la escasa información que se ha generado sobre las enfermedades y parásitos de la fauna silvestre en Baja California, en el estado de California, Estados Unidos de América (EE.UU.), existen muchos estudios sobre el tema. Dado que hay una continuidad biológica entre estos dos estados, dichos estudios pueden sugerir las enfermedades y parásitos a encontrar en Baja California. En un trabajo que cubrió la mayor parte del estado de California, se analizaron sueros de 279 venados cobrados por cazadores. En ellos se encontró prevalencia de anticuerpos de Anaplasma marginale (56\%), Borrelia burgdorferi (31\%), enfermedad de lengua azul (virus serotipo 17, 16\%), enfermedad viral hemorrágica epizoótica (15\%), Coxiella burnetii (7\%) y Toxoplasma gondii (7\%) (Chomel et al., 1994).

Nuestro estudio se enfocó en el venado bura (Odocoileus hemionus), el ungulado silvestre de más amplia distribución en Baja California (Mellink, 2005) y que comparte gran parte de su hábitat con ganado doméstico bovino, caprino, ovino y equino. Es importante conocer a qué parásitos y enfermedades está expuesto el venado, de manera que se puedan anticipar los posibles riesgos para la ganadería, la fauna silvestre y el hombre y planear acciones zoosanitarias adecuadas. El objetivo del presente estudio fue generar información sobre la presencia de dichas enfermedades y parásitos en venados cazados legalmente en el norte de la Sierra de San Pedro Mártir. 


\section{ÁREADEESTUDIO}

Las muestras se obtuvieron de venados de vida libre cobrados en la Unidad de Manejo y Conservación de Vida Silvestre (UMA) "El Tepi" (registro de la Secretaría de Medio Ambiente y Recursos Naturales; SEMARNAP UMA-EX-OO1- BC). La UMA El "Tepi" corresponde a los terrenos del Ejido del mismo nombre (71,000 hectáreas,

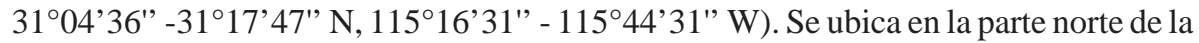
Sierra San Pedro Mártir y colinda al sur con este parque. Dado que la Sierra San Pedro Mártir cruza al Ejido Tepi de sur a norte, este tiene una topografía accidentada. Los puntos más altos dentro de los terrenos del ejido, alcanzan 2600 msnm, y se ubican en los límites del Parque Nacional San Pedro Mártir, y los más bajos (400 msnm) se encuentran en la Laguna Seca, Valle de Santa Clara. Debido a su fuerte gradiente topográfico, y además a que está dividido por el parteaguas peninsular, el ejido tiene cuatro tipos de clima (de acuerdo con la clasificación de Copen, modificado por E. García 1973); esencialmente desértico [(BW(h’)hw(x')] en el Este, mediterráneo [BSks], en el Oeste y dos tipos de clima templado (Cs y C(E)s(x') en el sur.

En concordancia con la variantes climáticas, existen cuatro tipos de vegetación en el ejido (SARH, 1991). El chaparral de montaña es un tipo de vegtacion con especies de plantas dominantes como Adenostoma sparcifolium, Adenostoma fasciculata y Yucca schidigera, principalmente en el centro norte y oeste del ejido (38.8\% de la superficie). En el matorral xerofilo predominan Prosopis sp., Larrea tridentata, y Opuntia spp. En la parte este del ejido (35.2\%), el bosque mixto esta compuesto de especies de coníferas como Pinus jeffreyi, P. quadrifolia, Juniperus californica y Adenostoma spp, en la parte centro sur (22.9\%) y Bosque de coníferas, donde predomina el Pinus jeffreyi, en la parte centro sur (1.7\%). Existen otras comunidades vegetales ocupando una porción muy pequeña de la superficie del ejido como las de arroyos perennes como el Río San Rafael y en pequeñas ciénegas, donde predominan especies como Populus sp., y Salíx sp. La principal actividad productiva del ejido ha sido la producción de crías de ganado. Sin embargo, la escasez de lluvias en los últimos años ha provocado la muerte y venta del ganado, quedando a la fecha menos de 200 cabezas de ganado bovino y 30 de caballar en el ejido. La actividad que actualmente proporciona mayores ingresos económicos a los ejidatarios es la cosecha de fustes de palmilla (Yucca schidigera), que se comercializa como materia prima industrial.

\section{MATERIALES Y MÉTODOS}

Los ejemplares de los que se tomaron las muestras fueron cobrados legalmente por cazadores durante las temporadas cinegéticas 2001-2002 al 2003-2004. A estos cazadores se les capacitó y dotó con un paquete de toma de muestras, que consistió 
en un par de guantes de látex; 1 jeringa estéril con capacidad de 10 mililitros y aguja calibre 21 G de 32 milímetros; 1 frasco de plástico estéril con capacidad de 100 mililitros para las muestras de excretas; 2 tubos estériles vacutainer de 5 mililitros, sin anticoagulante; 2 tubos estériles vacutainer de 5 mililitros, con anticoagulante; 1 tubo de plástico con capacidad de 10 mililitros conteniendo una solución de alcohol, agua y glicerina, para preservar ectoparásitos; 1 pluma y; 1 formato para toma de datos en campo.

Inmediatamente después de cobrarse un ejemplar, se tomó muestra de sangre del corazón y arterias principales, utilizando las jeringas de plástico estériles, y se depositó en los tubos estériles tipo «vacutainer,» con y sin anticoagulante (EDTA). De 15 muestras de sangre que se colectaron, 3 muestras se alteraron accidentalmente por hemólisis y no se pudieron analizar.

Se realizó una inspección ocular minuciosa de todo el cuerpo de cada venado cazado. Los ectoparásitos encontrados se colectaron manualmente y se colocaron dentro de los tubos con agua-alcohol-glicerina. Las excretas, se tomaron directamente del intestino grueso, entre los 20 y 50 centímetros anteriores al ano, después de eviscerar al ejemplar y abrir con navaja el intestino, y se depositaron en los contenedores estériles de plástico. Se tomó nota de la apariencia externa de los ejemplares y de sus órganos internos y en algunos casos se tomaron fotografías del proceso de toma de muestras. Todos los recipientes donde se colocaron las muestras se marcaron con la fecha de captura y el nombre del colector. Inmediatamente después de la toma de muestras se llenó el formato con información adicional.

Las muestras de sangre y excretas se guardaron en hieleras en las que se transportaron al laboratorio correspondiente. Siguiendo las recomendaciones de Chomel et al. (1994) y Couvillion (1980), las muestras de sangre se procesaron en menos de 36 horas después de su obtención, mientras que los sueros se guardaron en congelador a $-20^{\circ} \mathrm{C}$ grados centígrados, hasta su análisis. Las muestras de excretas se conservaron en hielo hasta su entrega al laboratorio, antes de 48 horas después de su colecta. Los ectoparásitos se entregaron al laboratorio para su identificación al mismo tiempo que las excretas.

Los ectoparásitos de las muestras 1 a la 9, se identificaron en el Laboratorio de Salud Animal de la Dirección General de Sanidad Animal, de la Secretaría de Agricultura, Ganadería, Desarrollo Rural, Pesca y Alimentación (SAGARPA, Km. 1.5 de la Carretera Mexicali a San Felipe, s/n, Col. Xochimilco, Mexicali, Baja California), donde se procesaron siguiendo los métodos señalados en la Norma Oficial Mexicana NOM-019-200-1994. Los ectoparásitos de las muestras 10 a la 15 se identificaron en el Laboratorio de Parasitología, del Instituto de Investigaciones en Ciencias Veterinarias de la Universidad Autónoma de Baja California (carretera a San Felipe Km 3, Fraccionamiento Campestre, Mexicali, Baja California) siguiendo el mismo método. Las excretas se analizaron en busca de evidencias de nemátodos endoparásitos en el 
Laboratorio de Salud Animal de la Dirección General de Sanidad Animal, mediante el método MacMaster.

Las muestras de sangre se analizaron en el Laboratorio de Diagnóstico de Tuberculosis y Brucelosis del Instituto de Investigaciones en Ciencias Veterinarias, de la Universidad Autónoma de Baja California. Para la detección de anticuerpos contra Anaplasma marginale se utilizó el paquete de identificación por ELISA competitivo , Anaplasma Antibody Test Kit cELISA (VMRD Inc. WA) tomando un valor mayor al 30\% de inhibición de la reacción de un anticuerpo monoclonal acoplado a la peroxidasa de rábano con el antígeno fijado en los pocillos de las placas de ELISA, como un caso seropositivo, tal como recomienda el proveedor; para detectar seropositividad a la enfermedad de lengua azul, se utilizó el paquete de identificación por ELISA competitivo Bluetongue Virus Antibody Test Kit cELISA (VMRD Inc. WA) tomando un valor mayor al 50\% de inhibición de la reacción de un anticuerpo monoclonal acoplado a la peroxidasa de rábano con el antígeno fijado en los pocillos de las placas de ELISA, como un caso seropositivo, también, tal como recomienda el proveedor; Para la detección de anticuerpos contra Brucelosis, se realizaron pruebas de aglutinación tarjeta y rivanol, de acuerdo con la NOM -041-200-1995 y con los procedimientos marcados en el manual de procedimientos de laboratorio INDRE/ SAGARPA (Hernández et al., 1996), utilizando el reactivo rosa de bengala y ABA TEST Rivanol de la Productora Nacional de Biológicos Veterinarios. Además se buscó ADN de Brucella sp. en testículos, mediante el método de PCR (Vemulapalli et al., 1999).

\section{RESULTADOS Y DISCUSIÓN}

\section{Ectoparásitos}

Se obtuvieron 9 especies de ectoparásitos, de cinco familias y dos clases taxonómicas, de los venados muestreados (Cuadro 1).

La garrapata hembra adulta del género Ixodes colectada fue identificada en el Laboratorio de Salud Animal, de la Dirección de Salud Animal en Mexicali, Baja California, y confirmada en el Centro Nacional de Servicios de Constatación en Salud Animal (SENASICA) el 5 de abril del 2003, como I. scapularis. Es extraña la identificación de I. scapularis en esta región, ya que esta es una garrapata ampliamente distribuida en el noreste, este y centro norte de los EE.UU., pero no en el oeste de Norteamérica, donde la única especie reportada es I. pacificus (Furman y Loomis, 1984; Dennis et al., 1998). Ambas garrapatas son muy similares. El ejemplar no se pudo recuperar para su verificación en otros laboratorios, por lo que no se puede definir si hubo una confusión, o si I. scapularis se ha introducido a la región, quizás por medio de movimientos de ganado, quedando por dilucidar esta cuestión en trabajos 
Cuadro 1. Ectoparásitos de machos de venados bura (Odocoileus hemionus) del Ejido Tepi, Baja California, México. 2001-2004.

\begin{tabular}{|c|c|c|c|}
\hline Familia & Especie & $\begin{array}{l}\text { Identificados Positivos } \\
\text { / Ejemplares muestreados }\end{array}$ & $\begin{array}{l}\text { Prevalencia } \\
\text { (\%) }\end{array}$ \\
\hline
\end{tabular}

Aracnidae: Acari

$\begin{array}{llcc}\text { Ixodidea } & \text { Ixodes sp. } & 3 / 15 & 20 \\ \text { Ixodidea } & \text { Rhipicephalus sanguineus } & 3 / 15 & 20 \\ \text { Ixodidea } & \text { Dermacentor occidentalis } & 11 / 15 & 73 \\ \text { Ixodidea } & \text { Dermacentor variabilis } & 1 / 15 & 6.67 \\ \text { Ixodidea } & \text { Dermacentor albipictus } & 3 / 15 & 20\end{array}$

Insecta: Diptera

$\begin{array}{llcc}\text { Hippoboscidae } & \text { Lipoptena sp } & 12 / 15 & 80 \\ \text { Oestridae } & \text { Hypoderma lineatum } & 1 / 15 & 6.67 \\ \text { Oestridae } & \text { Especie no identificada } & 1 / 15 & 6.67\end{array}$

Insecta: Anoplura

$\begin{array}{lll}\text { Haematopinidae } & \text { Linognathus vituli } & 1 / 15\end{array}$

posteriores. Estas dos especies de Ixodes pueden transmitir Enfermedad de Lyme y tularemia, e I. scapularis adicionalmente babesiosis, erliquiosis humana y anaplasmosis entre otras. Ixodes pacificus tambien puede transmitir ricketsias del grupo de las enfermedades maculosas, erliquiosis equina y se le implica en la transmisión de anaplasmosis (Allan, 2001b).

Ixodes scapularis tiene un amplio rango de hospederos, entre los que se pueden mencionar al venado cola blanca (Odocoileus virginianus), ardillas (Sciurus, Tamiasciurus), mapaches (Procyon lotor), zariguellas (Didelphys marsupiales), Zorras (Vulpes vulpes y Urocyon cinereargentatus), coyote (Canis latrans), lobo (Canis lupus), gato montés (Felis rufus), y animales domésticos como gato, ganado bovino, perro, cabra, caballos, mula, ovinos y porcinos, y el hombre, entre otros (Allan, 2001b)

Ixodes pacificus tiene muchos hospederos, incluyendo el venado bura (Odocoileus hemionus columbianus), ratones (Peromyscus spp, Perognathus spp y Zapus spp), ratas (Neotoma spp), ardillas (Spermophilus spp, Tamiasciurus spp y Eutamias spp), lobo (Canis lupus), puma (Puma concolor), gato montés (Lynx rufus), coyote (Canis latrans), lagomorfos (Sylvilagus spp y Lepus spp), musarañas y topos (Sorex spp y Scapanus spp), comadrejas (Mustela spp), fauna doméstica (burros, gatos, reses, perros, cabras, caballos y mulas) y el hombre (Allan, 2001b). Los estados inmaduros de esta especie se encuentran comúnmente sobre lagartijas de los géneros 
Gerrhonotus y Sceloporus, asi como en aves que habitan en el suelo y roedores pequeños y lagomorfos (Furman y Loomis, 1984). Esta garrapata es capaz de transmitir la enfermedad de Lyme, tularemia, erliquiosis y se le implica en la transmisión de anaplasmosis, erliquiosis equina y ricketsias del grupo de la fiebre maculosa de las Montañas Rocosas, y parálisis de las garrapatas (Allan, 2001b).

Rhipicephalus sanguineus tiene una distribución mundial, pero es más frecuente en climas templados. Es la garrapata más común en perros y en las infestaciones de casas (Rhodes y Norment, 1979). Esta garrapata es transmisora de varias enfermedades: Babesia canis, Ehrlichia canis, Anaplasma marginale, Hepatozoon canis, fiebre maculosa de las Montañas Rocosas, haemobartonellosis, tularemia, y parálisis de las garrapatas (Allan, 2001b).

Dermacentor occidentalis es una de las garrapatas más ampliamente distribuidas en California excepto en las áreas desérticas. Otras áreas donde se le ha colectado son los estados de Oregon, EE.UU. y Baja California, México (Furman y Loomis, 1984). Cuando es subadulto se alimenta de la sangre de roedores, especialmente ardillas, y como adulto de la de ganado bovino, equinos, venados y, raramente, el hombre. Es una garrapata vector de enfermedades zoonóticas como la tularemia, y es capaz de transmitir fiebre de Colorado (Allan, 2001b). También es vector de Anaplasma marginale) que tuvo una seropositividad de $56 \%$ en venados en el sur de California (Chomel et al., 1994).

Dermacentor variabilis se distribuye principalmente en el este de los Estados Unidos de Norteamérica (Kocan y Kocan, 1991; Allan, 2001b). Se reportó esta especie sobre los 12 borregos cimarrones cobrados durante los años 1976 y 1978, en las localidades Arroyo Grande, Matomí y Sierra San Pedro Mártir en Baja California (López, 1979). Este ectoparásito tiene muchos hospederos, con sus estados inmaduros alimentándose sobre pequeños mamíferos y sus adultos sobre mamíferos grandes. Se puede encontrar sobre animales domésticos como ganado bovino, perros, gatos, caballos, mulas, cerdos, borregos, así como sobre el hombre (Allan, 2001b). Esta especie es capaz de transmitir fiebre maculosa de las Montañas Rocosas, babesiosis, anaplasmosis, tularemia, fiebre Q y parálisis de las garrapatas (Allan, 2001b).

Dermacentor albipictus se distribuye en el norte, este y oeste de los EE.UU. y en Canadá. Es una especie que requiere de un solo hospedero para completar su ciclo biológico y solamente las hembras grávidas se desprenden y caen al suelo. Se alimentan principalmente sobre equinos y cérvidos, pero también sobre otros animales domésticos como ganado bovino, caballos, mulas, gatos y raramente sobre el hombre. Puede provocar parálisis de las garrapatas y se le implica en la transmisión de anaplasmosis (Allan, 2001b).

Las moscas Lipoptena sp. son ectoparásitos en su estado de adulto y se alimentan de sangre de sus hospederos, frecuentemente cérvidos, y Lipoptena depressa se encuentra generalmente sobre venado bura (Allan, 2001a). Existe poca 
información sobre los efectos que puedan causar a sus hospederos (Allan, 2001a), aunque Strickland et al. (1981) sugiere que infestaciones de Lipoptena podrían provocarles anemia o daños físicos.

Hypoderma lineatum parasita a diversos animales, incluyendo animales domésticos. En el ganado bovino, las larvas del último instar provocan tumores debajo de la piel, principalmente en el lomo (Borror y White, 1970). Cuando las moscas de esta especie se acercan al ganado a poner sus huevecillos, éste se pone nervioso y trata de escapar (Metcalf y Flint, 1979). Debido a que el ataque es persistente, los animales son constantemente irritados y no se alimentan suficientemente, lo que se refleja en una importante pérdida de peso y decremento en la producción de leche. Cuando son atacados por estas moscas, el ganado puede herirse solo y dañar su piel. Es posible que Hipoderma no alcance a completar su ciclo biológico en el venado, o que no sea común, pues en una consulta con cazadores que, en conjunto han cobrado más de 300 venados en Baja California ninguno recordó haber observado los síntomas ocasionados por larvas de esta especie.

Los únicos oestros que se han encontrado infestando venado buro en el oeste de Norteamérica son Cephenemya jellisoni y Cephenemya alicata (Hibler, 1981; vea también a Weber, 1992). Estos son Dípteros larvíparos que utilizan a los cérvidos como hospederos de sus fases larvales. Ocasionalmente estos parásitos llegan a ocasionar la muerte de sus hospederos, al penetrar la cavidad craneal (Johnson et al. en Coldwell (2001) pero, en general se han reportado pocos síntomas asociados con infestaciones (Colwell, 2001). Las infestaciones de este díptero sobre venado bura presentan una prevalencia que va del 25 al 95\%, dependiendo del año y las condiciones ambientales, y no es raro encontrar infestaciones fuertes, tanto sobre venados saludables, como sobre venados débiles (Hibler, 1981). En nuestro estudio no a todas las cabezas de los ejemplares se les revisaron los senos frontales y las bolsas retrofaríngeas.

Linognathus vituli es un piojo de distribución mundial (Durden, 2001). Uno de sus hospederos es el ganado vacuno, en el que infesta con mayor intensidad a los becerros, pero también se encuentra sobre venado. No se conocen sus efectos sobre los venados, pero sobre bovinos domésticos estos pequeños ectoparásitos pueden presentarse en miles o decenas de miles. Los piquetes que ocasionan estos piojos al alimentarse, provocan irritación, y el ganado infestado se ocasiona peladuras y golpes al frotarse con lo que encuentra para aliviar el ardor. Los animales afectados están predispuestos a ser atacados por otras enfermedades. La pérdida de sangre causada por este parásito puede provocar anemia y esta anemia causar abortos, y en algunas ocasiones de muy alta infestación este piojo llega a ocasionar la muerte (Mock, 1999). 


\section{Endoparásitos}

En las 15 muestras de excretas que se analizaron no se encontraron evidencias de nemátodos endoparásitos detectables por el método de Mac Master. Ello sugiere, en primera instancia, que las poblaciones de venado están por debajo de la capacidad de sustento del hábitat, ya que cuando se acercan a esta nivel aumentan sus cargas parasitarias, cuando menos del abomaso (Eve y Kellogs, 1977). Al mismo tiempo, no teniendo cargas endoparasitarias, los venados no representan un problema agropecuario potencial, en este contexto.

\section{Enfermedades}

Se detectaron anticuerpos contra la enfermedad de lengua azul y Anaplasma spp. , pero no contra Brucella abortus (Cuadro 2). La seroprevalencia de las enfermedades encontradas fue muy similar a la reportada para venados en California (Chomel et al., 1994). Anaplasma, con 56 \% de seroprevalencia en California y 66 \% en Baja California, y enfermedad de lengua azul, con $16 \%$ y $8.33 \%$, respectivamente.

Anaplasma marginale puede transmitirse entre el ganado doméstico y los venados por muchos ectoparásitos, incluyendo los que nosotros detectamos: Dermacentor albipictus, Dermacentor occidentalis, Dermacentor variabilis, Ixodes pacificus o Rhipicephalus sanguineus (Davidson y Goff, 2001). La anaplasmosis puede también estarse transmitiendo entre el ganado doméstico, venados) y borregos cimarrones por medio de la garrapata Dermacentor occidentalis, tal como se ha sugerido en otros sitios (Jessup et al., 1993), debido a que coexisten estas tres especies en partes del área de estudio.

Cuadro 2. Seroprevalencia de enfermedades en venado bura (Odocoileus hemionus) del Ejido Tepi, Baja California. 2001-2004.

Enfermedad Número de Positivas Negativas Sospechosas Seroprevalencia muestras

$\begin{array}{lllcrr}\text { Anaplasmosis } & 12 & 8 & 4 & 0 & 66.67 \% \\ \text { Lengua Azul } & 12 & 1 & 11 & 0 & 8.33 \% \\ \text { Brucelosis } & 12 & 0 & 12 & 1 & 0 \%\end{array}$


La enfermedad de lengua azul fue identificada positivamente en 1 muestra de 12 analizadas. Esta enfermedad es causa de preocupación, pues representa un riesgo para la fauna silvestre (Howert et al., 2001), incluyendo el venado cola blanca, el berrendo (Antilocapra americana) y el borrego cimarrón (Thorne et al. en Patton et al., 1994). Se piensa que la misma puede ser parcialmente responsable de la desaparición del borrego cimarrón de ciertas regiones de Texas (Robinson et al., 1967) y que puede ser una causa significativa de enfermedad en el venado cola prieta en California (Chomel et al., 1994; Jessup et al. en Patton et al., 1994).

El principal vector de esta enfermedad en Norteamérica es el mosquito Culicoides variipennis (Díptera: Ceratopogonidae; Thomas 1981). El virus causante de la enfermedad se replica en las glándulas salivales de este insecto, sin haber transmisión transovárica, por lo que se presume que el virus pasa el invierno (dado que no hay mosquitos) en los rumiantes (Valero, 1986). Culicoides spp. se encuentran ampliamente distribuidos, sobre todo donde se encuentran cuerpos de agua, ya que en su fase larvaria son acuáticos (Borror y White, 1970). En Baja California esta enfermedad podría transmitirse entre el venado y el borrego cimarrón, de manera similar a lo que ocurre en el sur de California (Bradley, 1992). Es también una enfermedad de alto riesgo para el ganado, y en Baja California, 76\% de 70 muestras de suero tomadas de ganado bovino fueron positivas a anticuerpos contra enfermedad de lengua azul (Stott et al., 1989).

En este estudio no encontramos seropositividad a Brucelosis. Sin embargo, dado que es una enfermedad muy importante para el ganado y para la salud humana y se encuentra presente en el ganado lechero del estado, es importante continuarse monitoreando en fauna silvestre, sobre todo en zonas de incidencia de la enfermedad en ganado bovino.

\section{CONCLUSIONES Y RECOMENDACIONES}

Encontrar en Baja California los mismos ectopitos y evidencias de dos enfermedades reportadas en California refleja la continuidad biológica entre ambos estados, por compartir parte de una misma región biogeográfica. Esto sugiere que puede encontrarse en Baja California otras enfermedades de alto riesgo no evaluadas aquí, como la enfermedad de Lyme y la bartonelosis, de las cuales se encuentran sus vectores y hospederos. Ambas pueden ocasionar serios problemas de salud humana. Es recomendable buscar evidencias de estas dos enfermedades en especies de Baja California. También es conveniente desarrollar un programa de capacitación básico dirigido al personal médico sobre el diagnóstico y tratamiento de estas enfermedades.

Se requieren más estudios para determinar si las enfermedades lengua azul y anaplasmosis están afectando a la población de venado en Baja California, ya que mediante este trabajo solamente se comprobó su seroprevalencia, pero no sus 
repercusiones poblacionales. También se requiere estudiar su comportamiento en el ganado doméstico y borrego cimarrón al existir en el hábitat vectores que son capaces de transmitir estas enfermedades entre ellos.

Los resultados indican que los venados tienen una carga de ectoparásitos y enfermedades que podrían estar compartiendo con el ganado doméstico que comparte su hábitat, ya que la mayor parte de los ectoparásitos encontrados sobre venado, también pueden usar como hospedero al ganado bovino, ovino, caprino, caballar, gatos y perros. Las evidencias de anaplasmosis y lengua azul en venado, significan que el ganado doméstico también está expuesto a ellas, por lo que pueden estarse registrando pérdidas de ganado, aunque no hay reportes publicados al respecto. El área de estudio de nuestro trabajo comprendió solo una pequeña fracción del estado de Baja California por lo que se requiere ampliar la investigación a otras áreas y especies de fauna silvestre.

\section{AGRADECIMIENTOS}

Este trabajo fue apoyado con recursos del Sistema de Investigación Estatal (Sistema Educativo del Estado de Baja California y del Consejo Nacional de Ciencia y Tecnología, proyecto 009 DA, del año 2002). Humberto Lafarga, Ricardo Ortíz, Jesús Alfonso Moraila, José León, José Pérez y Joaquín Contreras Domínguez ayudaron a colectar las muestras, Iván Lucero apoyó en el procesamiento de las muestras de sangre, Yolanda González Medina y Sergio Cueto ayudaron en la identificación de ectoparásitos. Gerardo Suzan y Manuel Weber hicieron numerosas aportaciones al escrito.

\section{LITERATURACITADA}

Aguirre A.A., R.G. McLean, R.S. Cook y T.J. Quan. 1992. Serologic Survey for Selected Arboviruses and Other Potential Pathogens in Wildlife from México. Journal of Wildlife Diseases, 28:435-442.

Allan, S.A. 2001a. Biting Flies (Class Insecta: Order Diptera). Pp. 18-45, en: Parasitic diseases of wild mammals. (W.M. Samuel , M.J. Pybus y A.A. Kocan, eds.) 2da ed. Iowa State University Press. Ames.

Allan, S.A. 2001b. Ticks (Class Arácnida: Order Acarina). Pp. 72-106, en: Parasitic diseases of wild mammals. (W.M. Samuel , M.J. Pybus y A.A. Kocan, eds.) 2da ed. Iowa State University Press. Ames.

Borror, D.J. y R.E. White. 1970. A field Guide to Insects. America north of Mexico. Peterson Field Guide Series. Houghton Mifflin. Boston.

Bradley, A.M., C.E. Dada. 1992. Spatial and Seasonal Distribution of Potential Vectors of Hemorrhagic Disease Viruses to Peninsular Bighorn Sheep in the Santa Rosa Mountains of Southern California. Journal of Wildlife Diseases, 28:192-205. 
Chomel, B.B., M.L. Carniciu, R.W. Castelli, T.M. Work y D.A. Jessup. 1994. Antibody prevalence of eight ruminant infectious diseases in California Mule and Black-Tailed deer (Odocoileus hemionus). Journal of Wildlife Diseases, 30:51-59.

Colwell, D.D. 2001. Bot Flies and Warble Flies (Order Diptera: Family Oestridae). Pp. 46-71, en: Parasitic diseases of wild mammals. (W.M. Samuel , M.J. Pybus y A.A. Kocan, eds.) 2da ed. Iowa State University Press. Ames.

Colodner, Ch.A. 2001. Evaluación del Estado de Salud de la Población de Borrego Cimarrón (Ovis canedensis cremnobates Elliot, 1903) en la Sierra San Pedro Mártir, Baja California, México. Tesis de Licenciatura en Biología. Benemérita Universidad Autónoma de Puebla. Puebla, México.

Couvillion, Ch.E., E.W. Jenney, J.E. Pearson y M.E. Coker.1980. Survey of Antibodies to viruses of Bovine Virus Diarrhea, Bluetongue, and Epizootic Hemorrhagic Disease in Hunter-Killed Mule Deer in New Mexico. JAVMA, 177:790-791.

Davidson, W.R. y W.L. Goff. 2001. Order Ricckettsiales. Pp. 455-477, en: Infectious Diseases of Wild Mammals. (S.E. Williams y I.K. Barker, eds.) 3ra ed. Iowa State Press. Ames.

Dennis, D.T., T.S. Nekomoto, J.C. Victor, W.S. Paul y J. Piesman. 1998. Reported distribution of Ixodes scapularis and Ixodes pacificus (Acari: Ixodidae) in the United States. Journal of Medical Entomology, 35:629-638.

Durden, L.A. 2001. Lice (Phthiraptera). Pp. 3-17, en: Parasitic diseases of wild mammals. (W.M. Samuel, M.J. Pybus y A.A. Kocan, eds.) 2da ed. Iowa State University Press. Ames.

Eve, J.H. y F.E. Kellogs. 1977. Management implications of abomasal parasites in southeastern white-tailed deer. Journal of Wildlife Management, 41:169-177.

Furman, D.P. y E.C. Loomis. 1984. The Ticks of California. Bulletin of the California Insect Survey, 25:1-239.

García E. 1973. Modificaciones al sistema de clasificación climática de Köppen. Universidad Nacional Autónoma de México. México, D.F.

Hernández M.I., F.G. Peña y X. Betancourt. 1996. Manual de Procedimientos de Laboratorio INDRE/SAGAR: Brucelosis. Instituto Nacional de Diagnóstico y Referencia Epidemiológicos. México, D.F.

Hibler, Ch.P. 1981. Diseases, en Mule and Black-Tailed of North America. Pp. 129-155, en: Mule and black-tailed deer of North America. (O.C. Wallmo, ed.) University of Nebraska Press.

Howert, E.W., D.E. Stallknecht y P.D. Kirkland. 2001. Bluetongue, Epizootic Hemorrhagic Disease, and other Orbivirus-Related Diseases. Pp. 77-97, en: Infectious Diseases of Wild Mammals. (S.E. Williams y I.K. Barker, eds.) 3ra ed. Iowa State University Press. Ames.

Jessup D.A., W.L. Goff, D. Stiller, M.N. Oliver, V.C. Bleich y W.M. Boyce. 1993. A retrospective serologic survey for Anaplasma spp. infection in three bighorn sheep (Ovis canadensis) populations in California. Journal of Wildlife Diseases, 29:547-554.

Kocan, A.A. y K.M. Kocan. 1991. Tick transmitted protozoan diseases of wildlife in North America. Bulletin of the Society Vector Ecology, 16:94-108.

López, F.M. 1979 Ecto and Endoparasites of the Desert Bighorn (Ovis Canadensis cremnobates) in Northern Baja California, Mexico. Desert Bighorn Council Transactions, 23:78. 
Mellink, E. 2005. El Venado Bura de Baja California. Pp. 353-362, en: Contribuciones Mastozoológicas en Homenaje a Bernardo Villa. (V. Sánchez-Cordero y R.A. Medellín, eds.). Instituto de Biología e Instituto de Ecología, UNAM, México, D.F.

Metcalf C.L. y W.P. Flint. 1979 Insectos destructivos e insectos útiles; sus costumbres y su control. Compañía Editorial Continental, S.A. México.

Mock, D.E, 1999. Lice on Beef Cattle. Pp. 1-4, en: Beef Cattle Handbook. MidWest Plan Service of Ames, Iowa State University, Ames.

Patton, J.F., T.M. Work, D.A. Jessup, S.K. Hietala, M.N. Oliver y N.J. Maciachian. 1994. Serologic Detection of Bluetongue Virus Infection of Black-Tailed Deer: Comparison of Serum Neutralization, Agar Gel Inmunodiffusion, and Competitive ELISA Assays. Journal of Wildlife Diseases, 30:99-102.

Rhodes, A.R. y E. Norment. 1979. Hosts of Rhipicephalus sanguineus (Acari: Ixodidae) in northern Mississippi, USA. Journal of Medical Entomology, 16:488-492.

Robinson R.M., T.L. Hailey, C.W. Livingston y J.W. Thomas. 1967. Bluetongue in the Desert Bighorn Sheep. Journal of Wildlife Management, 31:165-168.

SARH. 1991. Estudio para determinar el coeficiente de agostadero del Ejido Ganadero "Tep.", Comisión Técnica Determinadora de Coeficientes de Agostadero. Distrito de Desarrollo Rural 001, Ensenada B.C.

Stott J.L., M. Blanchard-Channell, B.I. Osburn, H.P. Riemann y R.C. Obeso. 1989. Serologic and Virologic Evidence of Bluetongue virus infection in Cattle and Sheep in México. American Journal of Veterinary Research, 50:335-340.

Strickland, R.K., R.R. Gerish y J.S. Smith. 1981. Arthropods. Pp. 363-389, en: Diseases and parasites of white tailed deer. (W.R. Davidson, F.A. Hayes, V.F. Nettles y F.F. Kellogg, eds.). Tall Timbers Research Station. Tallahassee, Florida.

Thomas, F.C. 1981. Hemorrhagic disease. Pp. 87-96, en: Diseases and parasites of withe-tailed deer. (W.R. Davidson, F.A. Hayes, V.F. Nettles, y F.E. Kellogg, eds.). Tall Timber Research Station Miscellaneous Publication Number 7, Tallahassee, Florida.

Valero, E.G. 1986. Lengua Azul. Pp. 355-358, en: Principales enfermedades de los ovinos y caprinos. (P. Pijoan y J. Tortora, eds.). Facultad de Estudios Superiores Cuautitlan, UNAM. México, D.F.

Vemulapalli R., J.R. McQuiston, G.G. Schurig, N. Sriranganathan, S.M. Halling y S.M. Boyle. 1999. Identification of an IS711 Element Interrupting the wboA Gene of Brucella abortus Vaccine Strain RB51 and a PCR Assay to Distinguish Strain RB51 from Other Brucella Species and Strains. Clinical and Diagnostic Laboratory Inmunology, 6:760-764.

Weber, M. 1992. Valoración clínica del efecto de la Ivermectina contra Cephenemyia spp. en venados cola blanca. Veterinaria México, 23:40-44. 